Review

\title{
Periodontal disease severity in subjects with dementia: A systematic review and meta-analysis
}

\author{
David Jonathan R. Gusman ${ }^{\mathrm{a}}$, João M. Mello-Neto ${ }^{\mathrm{a}}$, Breno Edson S. Alves ${ }^{\mathrm{a}}$, Henrique R. Matheus ${ }^{\mathrm{a}}$, \\ Edilson Ervolino ${ }^{\mathrm{b}}$, Letícia H. Theodoro ${ }^{\mathrm{a}}$, Juliano M. de Almeida ${ }^{\mathrm{a}, *}$ \\ ${ }^{a}$ Department of Surgery and Integrated Clinic, Division of Periodontics, São Paulo State University (Unesp), School of Dentistry, Araçatuba, São Paulo, Brazil \\ b Department of Basic Sciences, São Paulo State University (Unesp), School of Dentistry, Araçatuba, São Paulo, Brazil
}

\section{A R T I C L E I N F O}

\section{Keywords:}

Dementia

Meta-analysis

Periodontal disease

Systematic review

\begin{abstract}
A B S T R A C T
Background and objective: Despite clinical trials and reviews attempt to assess a possible relationship between dementia and periodontal disease, no meta-analysis has been performed and this issue remains undetermined. The aim of this study is to conduct a systematic review and meta-analysis to assess severity of periodontitis in subjects with dementia.

Methods: The search was conducted in Pubmed, Embase/MEDLINE. Two independent reviewers extracted data and assessed the risk bias (Newcastle-Ottawa scale). Meta-analyses were performed using the means of probing depth (PD) and clinical attachment loss (CAL) in patients with or without dementia. The mean difference were analyzed $(\mathrm{P} \leq 0.05)$.

Results: Fourteen studies were included in the systematic review. In the qualitative analysis, most studies reported higher prevalence of periodontal disease in dementia patients. The studies had low risk of bias and two meta-analyses were performed for each parameter, including or not a cross-sectional study. The meta-analyses including the cross-sectional study demonstrated significant association between dementia and periodontal disease (mean difference: $\mathrm{PD}=1.41$; $\mathrm{CAL}=1.40, \mathrm{P}<0.05$ ), however, it wasn't confirmed when the crosssectional study was removed $(1.25 \mathrm{~mm}, \mathrm{P}<0.22)$ and CAL $(1.20 \mathrm{~mm}, \mathrm{P}<0.22)$.

Conclusion: Although the qualitative analysis have suggested worse periodontal conditions in dementia patients, due to different study types and the high heterogeneity among them, the meta-analysis does not support the association between dementia and severity of periodontal disease.
\end{abstract}

\section{Introduction}

Dementia is a progressive syndrome, leading to a deterioration of cognitive function that can affect memory, orientation, the ability to perform routine work, behavior, thinking, understanding, learning ability, and judgment (Dementia, 2012). More than 47 million people suffer from dementia worldwide and 7.7 million new individuals are diagnosed with dementia every year. Moreover, by 2050, cases of dementia are estimated to increase to almost 115 million, three times the current estimate (World Alzheimer Report, 2009).

Dementia can be classified into different subtypes according to the associated brain pathologies, and the most common are Alzheimer's disease (AD), vascular dementia (VD), dementia with Lewy bodies (DLB), and frontotemporal dementia (FTD) (Chu, Ng, Chau, \& Lo, 2015). In 2016, it is estimated that 700,000 Americans aged $\geq 65$ years will die with Alzheimer's disease, and many of them will die because of the complications caused by Alzheimer's disease. These statistics underline the public health importance of identifying modifiable risk factors. Such data show that anything that contributes to the worsening of the patients' condition should be considered for treatment.

Futhermore, with the progression of severity in dementia, the ability of patients to perform self-care, including oral hygiene practice, deteriorates gradually (Chu et al., 2015), increasing the amount of bacterial plaque and debris, and resulting in inflammation and gingival bleeding (Gil-Montoya et al., 2015). Persistence of gingival inflammation is determinant for the development of periodontitis (Leask, Holmes, Black, \& Abraham, 2003; Schroeder, Munzel-Pedrazzoli, \& Page, 1973; Kurgan \& Kantarci, 2017) but may not consist an unquestionable statement to confirm progression of gingivitis to periodontitis (Kurgan \& Kantarci, 2017). The elements of host response and/or site-specificity shall be considered to explain long-term stability or progressive attachment loss in different sites in the same individual (Kurgan \& Kantarci, 2017).

\footnotetext{
* Corresponding autho at: Departamento de Cirurgia e Clinica Integrada,Disciplina de Periodontia, Faculdade de Odontologia de Araçatuba, UNESP, Rua José Bonifácio, 1193. CEP: 16015-050, Araçatuba,Sao Paulo, Brazil.

E-mail addresses: eervolino@foa.unesp.br (E. Ervolino), letheodoro@foa.unesp.br (L.H. Theodoro), julianomilanezi@foa.unesp.br (J.M. de Almeida).
} 
Considering these aspects, it is speculated that patients with dementia could have poor oral hygiene habits and worse periodontal condition. Therefore, the aim of this study is to conduct a systematic review and meta-analysis to assess the severity of periodontal disease in subjects with dementia.

\section{Methods}

This review is registered in the PROSPERO database (CRD42016053685), in compliance with the Preferred Reporting Items for Systematic Reviews and Meta-Analyses (PRISMA) statement guidelines (Moher, Liberati, Tetzlaff, Altman, \& PRISMA, 2010; Welch et al., 2012). The review also followed models proposed in the literature (Araújo et al., 2016; de Almeida et al., 2017).

\subsection{Literature search strategy}

Two independent reviewers (J.M.M.N., D.J.R.G.) conducted an electronic search on the PubMed/Medline and EMBASE databases for articles published in English within the last 20 years (January 1 st 1997 to September 2st, 2017). The key words used were: periodontal disease; dementia; Alzheimer's disease; vascular dementia; and frontotemporal dementia. Specifically; the PubMed search terms were as follows: $(((($ Periodontal disease and dementia)) OR (Periodontal disease and Alzheimer's disease)) OR (Periodontal disease and vascular dementia)) OR (Periodontal disease and frontotemporal dementia).

On Embase/MEDLINE, the literature search terms were: periodontal AND ('disease'/exp OR disease) AND ((''dementia'/exp OR dementia OR alzheimers) AND ('disease'/exp OR disease) OR vascular) AND ('dementia'/exp OR dementia) OR frontotemporal) AND ('dementia'/exp OR dementia) AND [english]/lim AND ([embase]/lim OR [medline]/ lim) AND [1997-2017]/py.

A further manual search was conducted of the reference lists of relevant review studies. We reviewed all potential abstracts and complete texts, and selected those that met the criteria detailed below. Disagreements between researchers were settled by consensus. Cohen's kappa coefficient was used to evaluate the disagreement between the researchers.

\subsection{Focus question}

In accordance with the PICO framework (Miller \& Forrest, 2001), we used the focus question "Is there an association between dementia and periodontal disease severity in older dementia adults?"

- Population: patients with dementia and without dementia, based on case definitions used in the publications

- Intervention: different periodontal indexes (PI: Plaque index; BOP: Bleeding on probing; GBI: Gingival bleeding index; PD: Probing depth; CAL: Clinical attachment loss; CPI: Community Periodontal index; CPITN: Community Index of Periodontal Treatment Needs (Ainamo et al., 1982; Ainamo \& Bay, 1975; Cestari et al., 2016; Dhingra \& Vandana, 2011; Gil-Montoya, Sánchez-Lara et al., 2017; Gil-Montoya, Barrios et al., 2017; Haffajee \& Socransky, 1986; Joss, Adler, \& Lang, 1994; Listgarten, 1980; Martande et al., 2014; Newbrun, 1996; Rai, Kaur, \& Anand, 2012; World Health Organization, 2005) (Table 1)

- Comparison: between the dementia and non-dementia group through the periodontal indexes presented

- Outcomes: Poorer results in the periodontal indexes in the dementia patients

\subsection{Inclusion criteria}

Case-control, cross-sectional, longitudinal and cohort studies in humans with at least six patients that evaluated periodontal indexes in patients with dementia, with mild cognitive impairment, and without dementia.

\subsection{Exclusion criteria}

Studies that did not evaluate periodontal indexes or evaluated only one periodontal index, without control groups, with only abstract available and those without access (Bramanti et al., 2015; Chen, Wu, \& Chang, 2017; Chalmers, Carter, \& Spencer, 2003; Fereshtehnejad et al., 2017; Hatipoglu, Kabay, \& Güven, 2011; Kamer et al., 2009; Kim et al., 2007; Lee, Lee et al., 2017; Lee, Hu, Huang, Chou, \& Chu, 2017; Luo et al., 2015; Noble, Manly, Schupf, Tang, \& Luchsinger, 2012; Noble et al., 2014; Noble et al., 2017; Sochocka et al., 2017a; Sochocka et al., 2017b; Singh, 2016; Sparks Stein et al., 2012; Stewart et al., 2015; Takeuchi et al., 2017; Zenthöfer, Meyer-Kühling et al., 2016) (studies were considered without access after sending an e-mail to their corresponding authors) (Table 2).

\subsection{Included in meta-analyses}

We included studies that had, as variables, PD and CAL that used parametric data (means) in patients with and without dementia in a subsequent meta-analysis (Cestari et al., 2016; Gil-Montoya et al., 2015; Rai et al., 2012; Martande et al., 2014).

\subsection{Assessment of bias within studies}

The qualities of the studies selected for the meta-analysis were evaluated based on the Newcastle-Ottawa scale (NOS) (You, Qu, \& Yu, 2016; Wells et al., 2011). The scale has been shown to be reliable and valid (Li et al., 2008). Studies scoring five or more points were considered to be of high quality (Higgins \& Thompson, 2002).

\subsection{Statistical analysis}

The Comprehensive Meta-Analysis Program (Review Manager 5.3, Cochrane Collaboration, Oxford, UK) was used for the meta-analysis. The meta-analysis was conducted including studies with similar comparisons and the same outcomes.

The fixed effects model was used when there was no statistically significant difference, and the random effects model was adopted when there was a statistically significant difference, which means a high index of heterogeneity among the trials. Heterogeneity was considered significant when $p<0.01$. Heterogeneity was assessed using a method in which the $\chi^{2}$ and $\mathrm{I}^{2}$ values were measured. The statistical value of $\mathrm{I}^{2}$ was used to analyze variations in heterogeneity; values above $75 \%$ (0-100\%) indicate relevant heterogeneity (Atieh, Ibrahim, \& Atieh, 2010; Annibali et al., 2012; Higgins \& Thompson, 2002). In cases with no appropriate data, the values were calculated.

\section{Results}

The electronic search on the databases identified 440 articles (Fig. 1 shows details of the research process and the study selection). After elimination of duplicates and analyses of the titles and abstracts according to the inclusion and exclusion criteria, we considered 34 articles as eligible; however, we had no access to the study by Bramanti et al. (Bramanti et al., 2015) which was excluded. Thus, 33 full texts were analyzed, and 19 were excluded based on the criteria (Table 2).

Finally, 14 articles were selected for systematic review (Chen, Shuman, Hodges, Gatewood, \& Xu, 2010; Chen, Clark, \& Naorungroj, 2013; Chu et al., 2015; Cestari et al., 2016; De Souza Rolim et al., 2014; Gil-Montoya et al., 2015; Gil-Montoya, Sánchez-Lara et al., 2017; GilMontoya, Barrios et al., 2017; Martande et al., 2014; Okamoto et al., 2010; Rai et al., 2012; Syrjälä et al., 2012; Zenthöfer, Cabrera, Rammelsberg, \& Hassel, 2016; Zenthöfer et al., 2017) (Table 3), with 
Table 1

Periodontal indexes evaluated in the intervention criteria.

Plaque index (PI)
Bleeding on probing (BOP)
Gingival bleeding index (GBI)
Probing depth (PD)
Clinical attachment loss (CAL)
Community index of periodontal treatment needs
$\quad$ (CPITN)

\author{
Mean (Rai et al., 2012; Martande et al., 2014; Gil-Montoya, Sánchez-Lara et al., 2017; Gil-Montoya, Barrios et al., 2017) \\ or percentage (Cestari et al., 2016) of bacterial plaque in the cervical region of the teeth. \\ Presence or absence of bleeding after insertion of periodontal probe within the sulcus or pocket (Joss et al., 1994). \\ Presence or absence of bleeding after gentle probing of the orifice or the gingival crevice (Ainamo \& Bay, 1975; Newbrun, \\ 1996). \\ Distance from the gingival margin to the base of the sulcus or pocket (Listgarten, 1980). \\ Distance from the cemento-enamel junction to the base of the sulcus or pocket (Haffajee \& Socransky, 1986). \\ CPITN assesses the presence or absence of gingival bleeding on probing, supra or subgingival calculus and periodontal \\ pockets by using a $0.5 \mathrm{~mm}$ ball tip WHO probe. Ten index teeth are examined, resulting in six scores determining the \\ individual's treatment needs (Ainamo et al., 1982; Dhingra \& Vandana, 2011). \\ Score 0: health periodontal conditions \\ Score 1: gingival bleedings \\ Score 2: calculus and bleeding \\ Score 3: shallow periodontal pockets (4 to 5 millimeters) \\ Score 4: deep periodontal pockets (6 millimeters or more) \\ Score $\mathrm{X}$ : When only one or no teeth are present in a sextant \\ CPI is a modified version of CPITN by inclusion of measurement of 'Loss of attachment' and elimination of 'Treatment \\ needs'. The periodontal status is assessed with a $0.5 \mathrm{~mm}$ ball tip WHO probe taking into consideration 10 teeth in the oral \\ cavity. The scores are: \\ Score 0: health periodontal conditions \\ Score 1: gingival bleedings \\ Score 2: calculus and bleeding \\ Score 3: shallow periodontal pockets (4 to 5 millimeters) \\ Score 4: deep periodontal pockets (6 millimeters or more) (Dhingra \& Vandana, 2011; World Health Organization, 2005)
}

Table 2

List of excluded studies according to the exclusion criteria and access availability.

\begin{tabular}{|c|c|}
\hline No control groups & $\begin{array}{l}\text { Zenthöfer et al. (Zenthöfer, Meyer-Kühling et al., 2016); Sochocka et al. (Sochocka et al., 2017a), Lee et al. (Lee, Hu } \\
\text { et al., 2017) }\end{array}$ \\
\hline Evaluates only plaque index (PI) & Chalmers et al. 2003 (Chalmers et al., 2003), Hatipoglu et al. 2011 (Hatipoglu et al., 2011) \\
\hline $\begin{array}{l}\text { Didn't evaluate the clinical parameters of } \\
\text { periodontal disease }\end{array}$ & $\begin{array}{l}\text { Kim et al. (Kim et al., 2007), Kamer et al. (Kamer et al., 2009), Noble et al. (Noble et al., 2012), Sparks Stein et al. } \\
\text { (Sparks Stein et al., 2012), Noble et al. (Noble et al., 2014), Luo et al. (Luo et al., 2015), Stewart et al. (Stewart et al., } \\
\text { 2015), Lee et al. (Lee, Lee et al., 2017), Fereshtehnejad et al. (Fereshtehnejad et al., 2017), Chen et al. (Chen et al., } \\
\text { 2017), Takeuchi et al. (Takeuchi et al., 2017) }\end{array}$ \\
\hline Only abstract available (Congress Presentation) & Noble et al. (Noble et al., 2017), Sochocka et al. (Sochocka et al., 2017b), Singh (Singh, 2016) \\
\hline No access & Bramanti et al. (Bramanti et al., 2015) \\
\hline
\end{tabular}

four of them being included in the meta-analyses (Gil-Montoya et al., 2015; Rai et al., 2012; Martande et al., 2014; Cestari et al., 2016) (Fig. 2a, b, c, d). The Kappa test statistic was 1.0 for the studies analyzed, indicating no disagreement between the reviewers.

\subsection{Qualitative review of studies}

Among the studies comparing the periodontal indexes between the dementia group and the non-dementia group, we found 1750 individuals with dementia (general mean age: 78.16 ) and 4816 without dementia (general mean age: 75.45). One study assessed only mild memory impairment (Okamoto et al., 2010), seven studies assessed dementia without distinguishing its type or degree of cognitive impairment (Chen et al., 2010; Chen et al., 2013; De Souza Rolim et al., 2014; Gil-Montoya, Sánchez-Lara et al., 2017; Gil-Montoya, Barrios et al., 2017; Rai et al., 2012; Zenthöfer, Cabrera et al., 2016; Zenthöfer et al., 2017), one study divided their case groups according to types of dementia (Syrjälä et al., 2012), three studies evaluated only Alzheimer's disease (Chu et al., 2015; De Souza Rolim et al., 2014; Martande et al., 2014), one study evaluated mild cognitive impairment and dementia of any type or severity, together in the same group (Gil-Montoya et al., 2015), one study evaluated dementia severity (Gil-Montoya, SánchezLara et al., 2017), and one study had Alzheimer's disease and mild cognitive impairment in different case groups (Cestari et al., 2016).

In general, most of these studies showed that periodontal disease was more prevalent in patients with dementia (regardless of the type of dementia) than those without. The values for PI, BOP, PD and CAL indicated a larger prevalence of periodontal disease in the dementia group (Cestari et al., 2016; Gil-Montoya et al., 2015; Gil-Montoya, Barrios et al., 2017; Gil-Montoya, Sánchez-Lara et al., 2017; Rai et al.,
2012; Martande et al., 2014). In addition, the severity of periodontal disease was found to worsen in accordance with the progression of Alzheimer's disease from mild to moderate and severe (Gil-Montoya, Sánchez-Lara et al., 2017; Martande et al., 2014). CPI and CPITN indicated greater need for periodontal treatment in dementia patients (Okamoto et al., 2010; Zenthöfer, Cabrera et al., 2016).

Some studies evaluated by this systematic review (Chu et al., 2015; Chen et al., 2010; Okamoto et al., 2010) reported worsen periodontal indexes in initial periods of control and in late periods of dementia groups. Moderate periodontitis was shown to be more prevalent in the initial stages in the control group, while the severe stages of periodontitis were found to be more prevalent in the dementia group (De Souza Rolim et al., 2014).

Only three studies did not find a significant difference between periodontal disease and dementia (Chu et al., 2015; Cestari et al., 2016; Syrjälä et al., 2012) and Zenthofer et al (Zenthöfer et al., 2017) did not find a statistical difference in GBI between the groups.

\subsection{Risk of bias assessment}

All the studies included in this meta-analysis (Cestari et al., 2016; Gil-Montoya et al., 2015; Martande et al., 2014; Rai et al., 2012) are considered to be of high quality according to the NOS scores (Luo et al., 2015).

\subsection{Results of the meta-analysis}

The meta-analyses were performed based on two different clinical parameters (PD and CAL), with (Fig. 2a, b) and without (Fig. 2c, d) cross-sectional study (Martande et al., 2014). For Martande et $\mathrm{al}^{14}$ and 


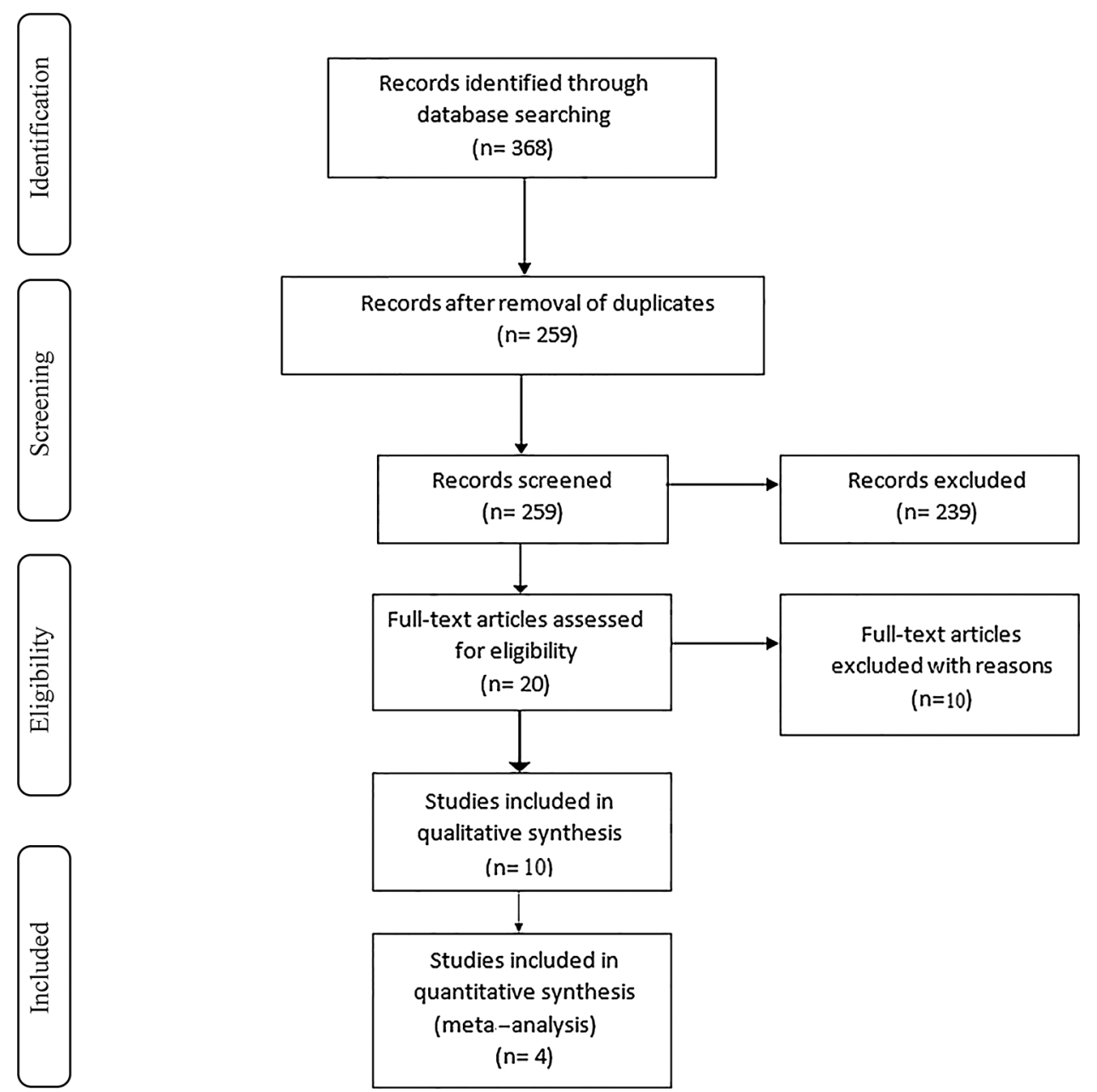

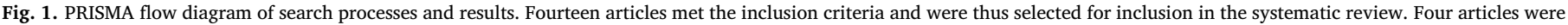
included in the meta-analysis.

Cestari et al, (Cestari et al., 2016) the means needed to be calculated due to the division in their case groups (mild, moderate, and severe Alzheimer's disease, and Alzheimer's disease and mild cognitive impairment). The meta-analyses had high statistical and clinical heterogeneity (PD: both $\mathrm{I}^{2}=98 \%$; CAL: both $\mathrm{I}^{2}=99 \%$ ).

In a random effects analysis including the study by Martande et al, (Martande et al., 2014) the PD was significantly higher in the dementia group compared to the control group, regardless of the level of severity of dementia $(1.41 \mathrm{~mm}, \mathrm{P}<0.01)$ (Fig. 2a). The CAL was also significantly higher in the group with dementia than in the control group, regardless of the level of severity of dementia $(1.40 \mathrm{~mm}, \mathrm{P}<0.01)$ (Fig. 2b)

The meta-analyses without the cross-sectional study ${ }^{14}$ showed no statistical difference in PD $(1.25 \mathrm{~mm}, \mathrm{P}<0.22)$ (Fig. 2c) and CAL $(1.20 \mathrm{~mm}, \mathrm{P}<0.22)$ (Fig. 2d) between the dementia and control group.

\section{Discussion}

Impairment of cognitive capabilities and daily life activities predisposes dementia patients to poor oral health and poor oral hygiene (Syrjälä et al., 2012). Furthermore, as the world's population ages and dementia becomes more prevalent, it will possibly become a public health problem (Dementia, 2012). In response to the clinical needs of an aging population and the resultant growing incidence of dementia, this study was conducted to assess the severity of periodontal disease in subjects with dementia compared to non-dementia patients.

Oral health plays a key role in the prevention of several diseases, especially in older adults (Gil-Montoya et al., 2015), making extremely important the knowledge concerning the periodontal conditions of dementia patients and how these conditions can affect people's daily lives. Patients with dementia typically have difficulties regarding plaque control, such as an opposition to oral care, and forgetting to brush their teeth, which increase the risk of developing periodontal disease (Chalmers et al., 2003; Chen et al., 2013).

Chalmers et $\mathrm{al}^{29}$ affirmed that these patients considerably worsen their oral conditions in one year, possibly because of the progressive neurodegeneration in this period. Martande et $\mathrm{al}^{14}$ also showed that the more severe the dementia, the more serious the involvement by periodontal disease.

Although the initial stages of periodontal disease were more prevalent in the control group in some studies, the severe periodontal disease was found to be more prevalent in the dementia group in several studies. (Chu et al., 2015; Chen et al., 2013; De Souza Rolim et al., 2014; Gil-Montoya, Sánchez-Lara et al., 2017; Gil-Montoya, Barrios et al., 2017; Okamoto et al., 2010) These findings suggest that periodontal disease and its progression may be associated with the presence and evolution of dementia; however, there is not yet enough data to 


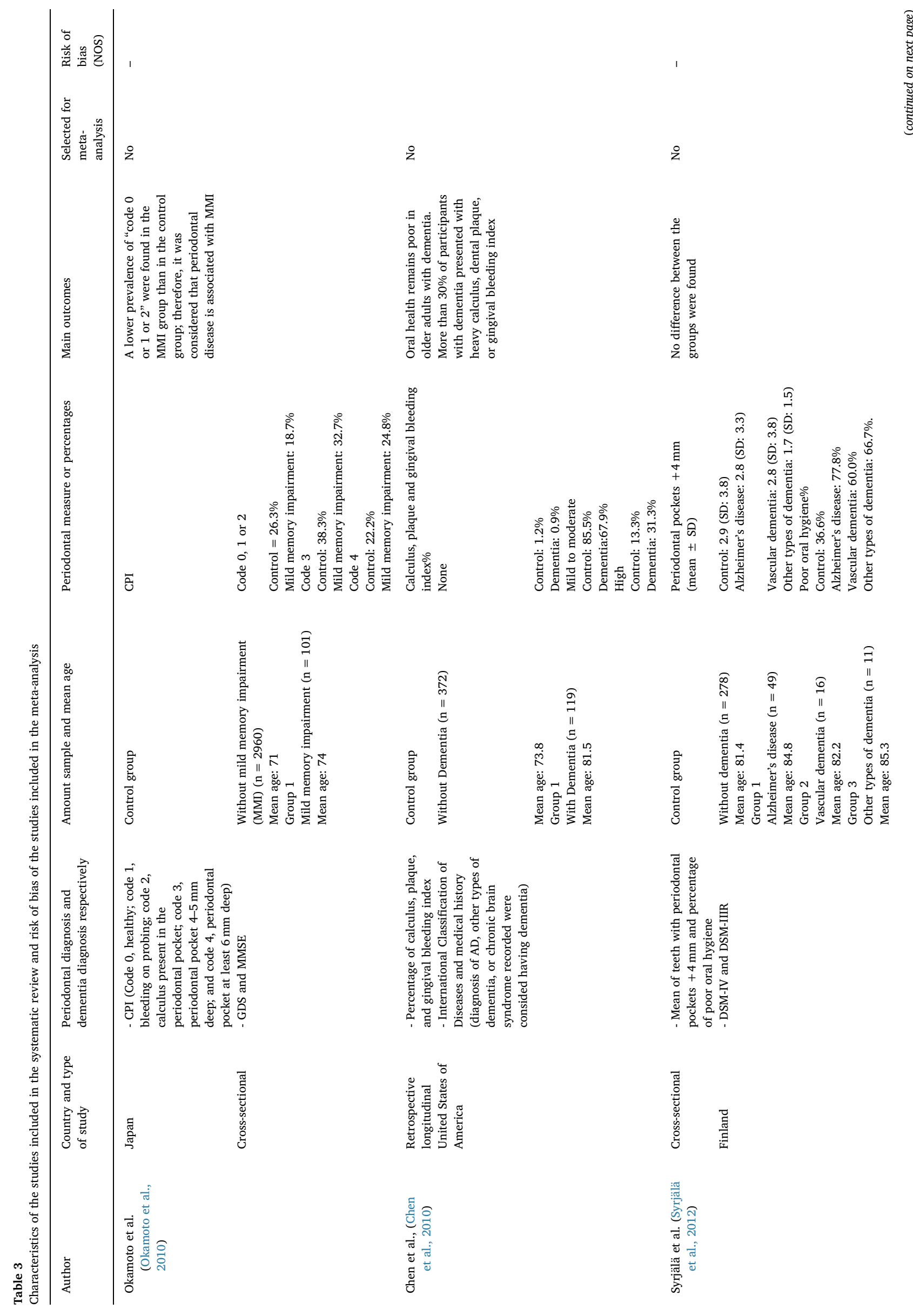




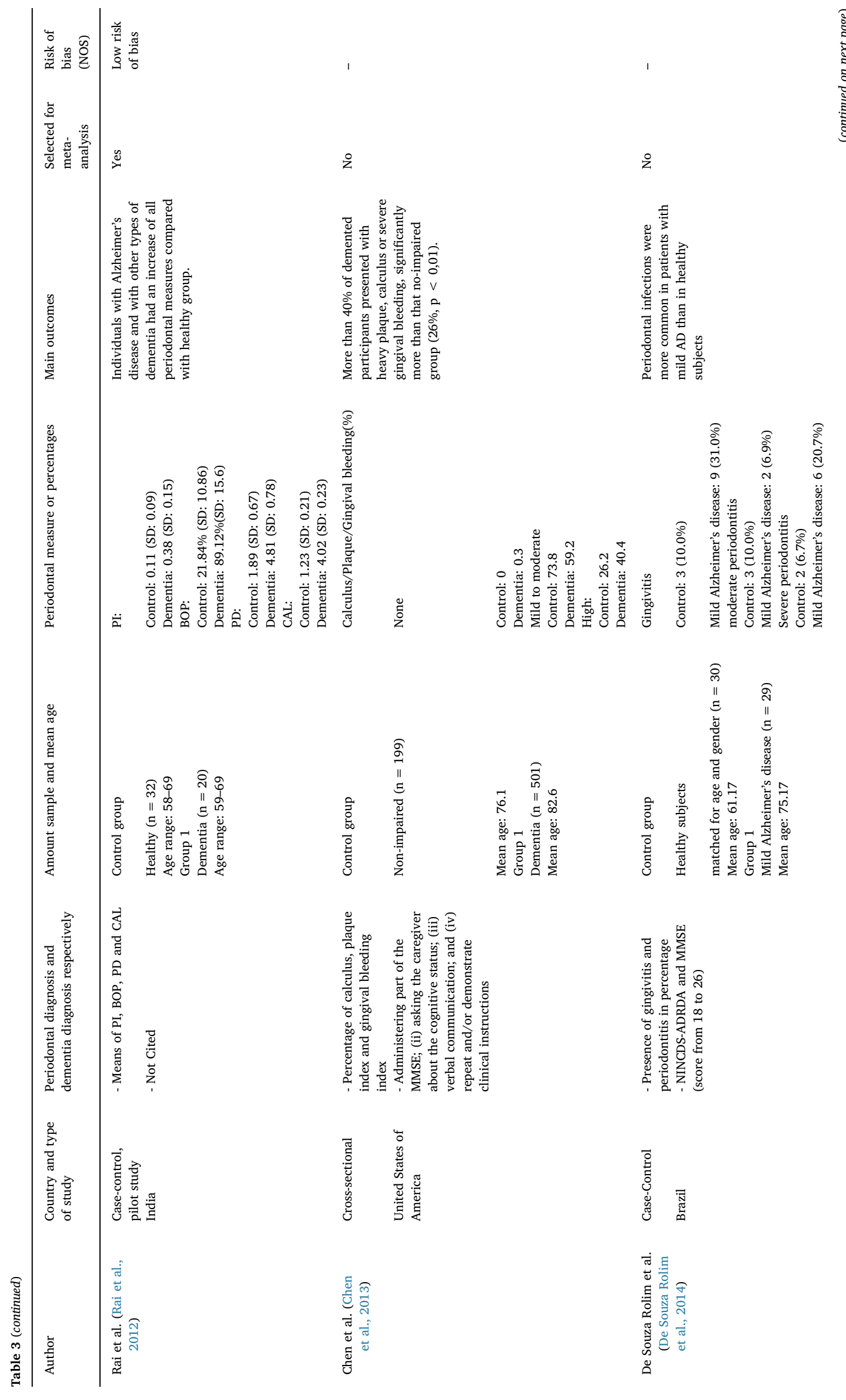




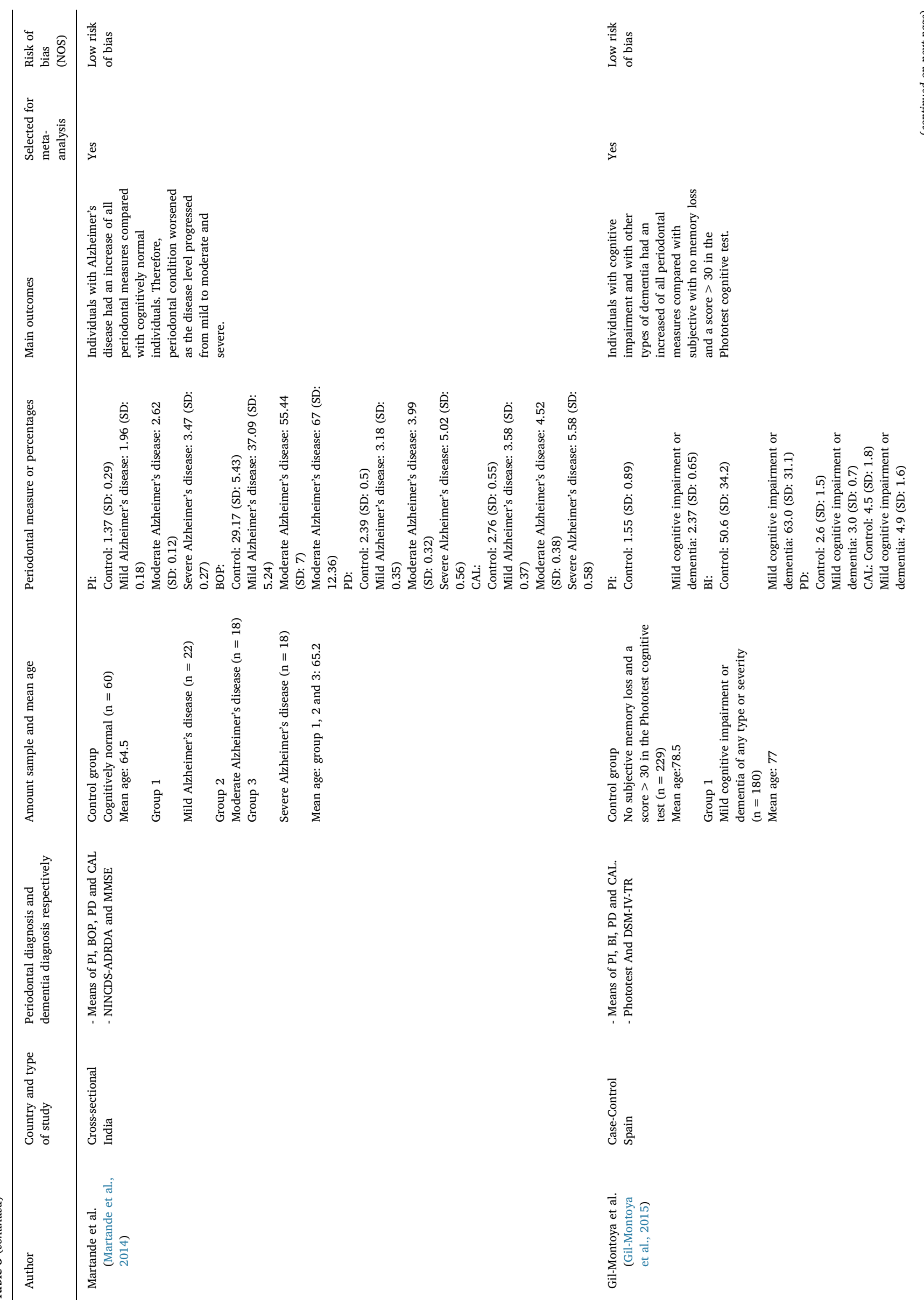




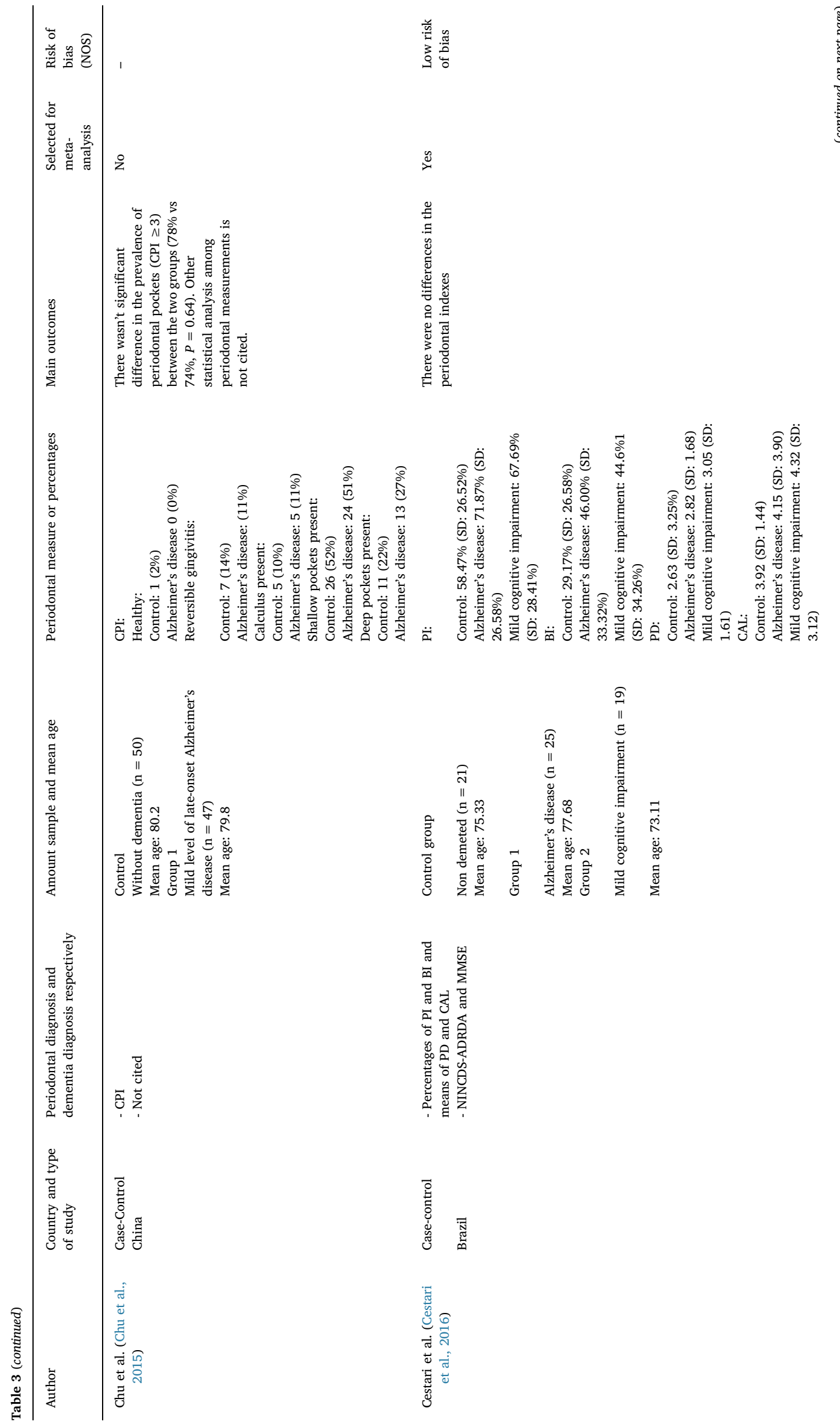




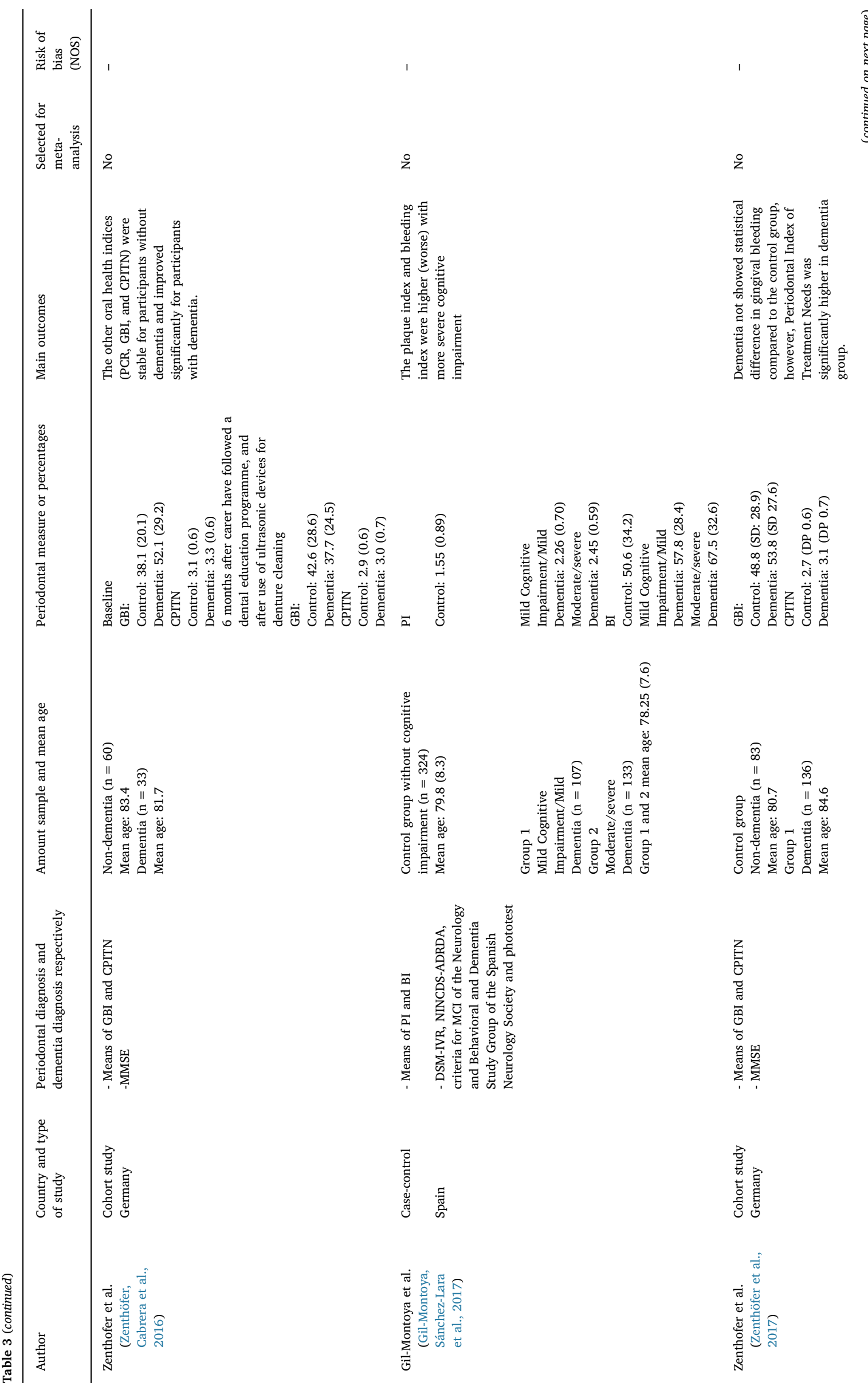




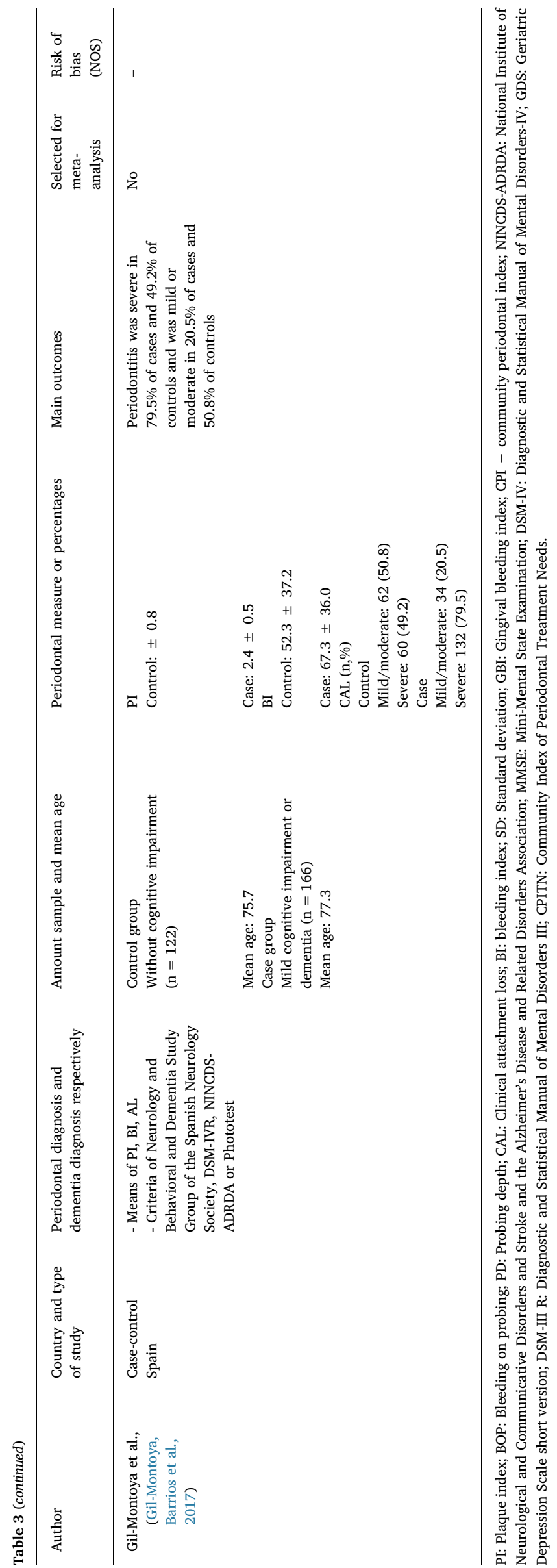

prove this hypothesis.

The present study is the first meta-analysis to assess the severity of periodontal disease in non-dementia versus dementia patients. PD and CAL are important clinical parameters to indicate the presence of periodontitis. Our study showed that PD and CAL were significantly higher in the dementia group compared to the control group regardless of the severity level of dementia $(\mathrm{PD}=1.41 \mathrm{~mm}, \mathrm{P}<0.01$; Fig. 2a) $(\mathrm{CAL}=1.40 \mathrm{~mm}, \mathrm{P}<0.01$; Fig. $2 \mathrm{~b})$. However, is important to emphasize that in cross-sectional studies, it is not always possible to establish a causal link, since exposure and disease are evaluated at the same moment, increasing the risk of bias. Therefore, despite the significant increase of PD and CAL highlighted by the meta-analyses including the cross-sectional study, the meta-analyses without the crosssectional study showed no association between dementia and periodontal disease severity $(\mathrm{PD}=1.25 \mathrm{~mm}, \quad \mathrm{P}<0.22 ; \quad$ Fig. $2 \mathrm{c})$ $(\mathrm{CAL}=1.20 \mathrm{~mm}, \mathrm{P}<0.20$; Fig. $2 \mathrm{~d})$. The same plausible tendency was reported by Pazos et al (Pazos et al., 2016) and Wu et al. (Wu et al., 2016)

Other parameters such as CPI, PI, BOP, and CPITN were not statistically analyzed due to the inclusion criteria of the meta-analysis; however, all these clinical parameters were higher in the groups with some type of dementia compared to the control group (Chen et al., 2010; Chen et al., 2013; De Souza Rolim et al., 2014; Gil-Montoya et al., 2015; Okamoto et al., 2010; Rai et al., 2012).

A limitation faced by the present study was the lack of details concerning the type of dementia and severity of AD. However, every type of dementia and $\mathrm{AD}$ showed worse results compared to the control groups. This finding suggests that patients with dementia need help in daily oral self-care and that, moreover, relatives, caregivers, and nurses in nursing homes and hospitals should be informed and instructed to help meet this requirement (Syrjälä et al., 2012). Second, the methodological approaches used in the studies were different, and several different instruments, such as the Geriatric Depression Scale - short version (GDS), Mini-Mental State Examination (MMSE), Diagnostic and Statistical Manual of Mental Disorders-IV (DSM-IV), Diagnostic and Statistical Manual of Mental Disorders III (DSM-III), National Institute of Neurological and Communicative Disorders and Stroke and the Alzheimer's Disease and Related Disorders Association - Alzheimer's Criteria (NINCDS-ADRDA Alzheimer's Criteria), Phototest, and medical history were used to determine dementia. There was also a large variation in sample size among the studies. These limitations may be responsible for the conflicting results among some studies, and the high statistical and clinical heterogeneity in both meta-analyses, both with (PD: $\mathrm{I}^{2}=98 \%$; CAL: $\mathrm{I}^{2}=99 \%$ ) and without the cross-sectional study (PD: $\mathrm{I}^{2}=98 \%$; CAL: $\mathrm{I}^{2}=99 \%$ ). The results presented by clinical trials shall be interpreted with caution, once statistically significant differences may not always represent clinical significance.

In summary, dementia patients might be less collaborative with oral hygiene and this can adversely affect their periodontal condition, though the association between dementia and the severity of periodontal disease remains unclear. Although worse periodontal conditions to be found in patients with dementia, the study of Chen et al. (Chen et al., 2010) affirmed that routine dental care carried by caregivers and dental professionals was capable to improve periodontal indices until reaching clinical outcomes similar to those without dementia, highlighting the reversibility of worse periodontal conditions and emphasizing the necessity of assisted dental care and professional assistance for dementia patients.

In conclusion, although the qualitative analysis have suggested worse periodontal conditions in dementia patients, due to different study types and the high heterogeneity among them, the meta-analysis does not support the association between dementia and severity of periodontal disease. Therefore, further research is needed to clarify this association. 


\section{With cross-sectional study}

(a) Probing depth

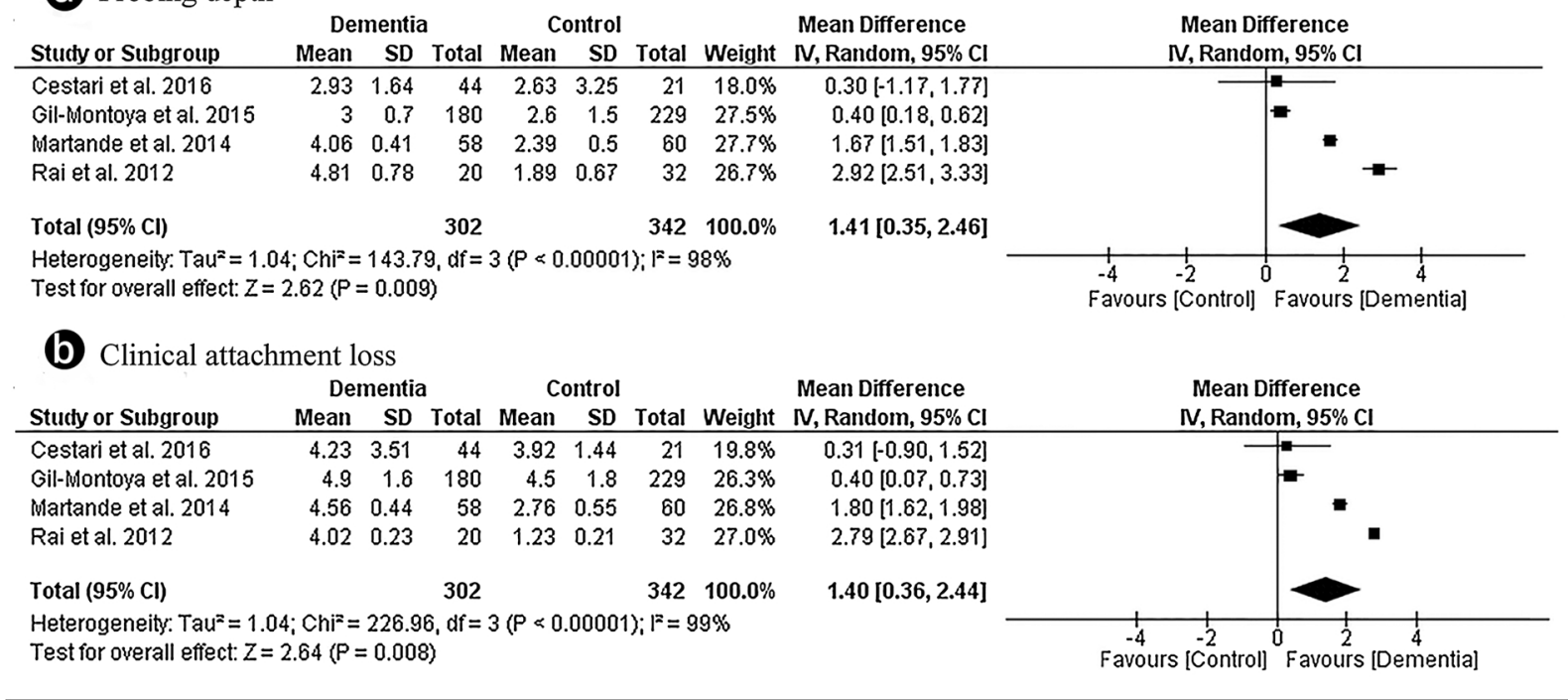

\section{Without cross-sectional study}

C Probing depth

\begin{tabular}{|c|c|c|c|c|c|c|c|c|c|c|c|}
\hline \multirow[b]{2}{*}{ Study or Subgroup } & \multicolumn{3}{|c|}{ Dementia } & \multicolumn{3}{|c|}{ Control } & \multicolumn{2}{|r|}{ Mean Difference } & \multirow{2}{*}{\multicolumn{2}{|c|}{$\begin{array}{l}\text { Mean Difference } \\
\mathrm{N}, \text { Random, } 95 \% \mathrm{Cl}\end{array}$}} & \\
\hline & Mean & SD & Total & Mean & SD & Total & Weight & IV, Random, $95 \% \mathrm{Cl}$ & & & \\
\hline Cestari et al., 2016 & 2.93 & 1.64 & 44 & 2.63 & 3.25 & 21 & $29.7 \%$ & $0.30[-1.17,1.77]$ & & & \\
\hline Gil-Montoya et al., 2015 & 3 & 0.7 & 180 & 2.6 & 1.5 & 229 & $35.3 \%$ & $0.40[0.18,0.62]$ & & 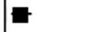 & \\
\hline Rai et al., 2012 & 4.81 & 0.78 & 20 & 1.89 & 0.67 & 32 & $35.0 \%$ & $2.92[2.51,3.33]$ & & & \\
\hline Total $(95 \% \mathrm{Cl})$ & & & 244 & & & 282 & $100.0 \%$ & $1.25[-0.74,3.24]$ & & & \\
\hline \multicolumn{9}{|c|}{$\begin{array}{l}\text { Heterogeneity: } \text { Tau }^{2}=2.90 ; \mathrm{Cni}^{2}=112.17, \mathrm{df}=2(\mathrm{P}<0.00001) ; \mathrm{I}^{2}=98 \% \\
\text { Test for overall effect: } Z=1.23(\mathrm{P}=0.22)\end{array}$} & $\begin{array}{lll}-4 & -2 & 0 \\
\text { Favours [Control] }\end{array}$ & Favours [ & $\begin{array}{c}4 \\
4\end{array}$ \\
\hline
\end{tabular}

d Clinical attachment loss

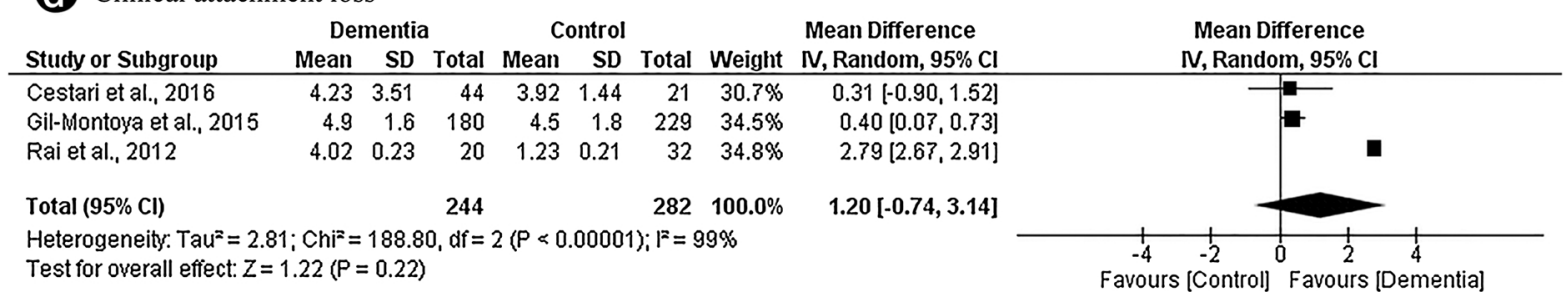

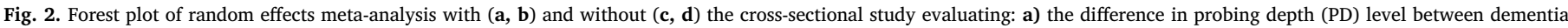

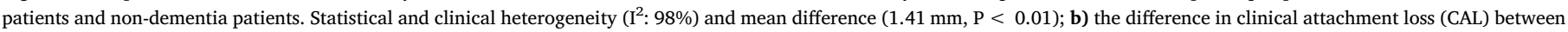

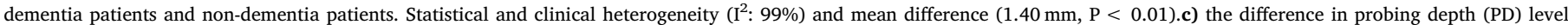

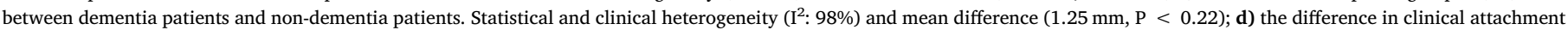
loss (CAL) between dementia patients and non-dementia patients. Statistical and clinical heterogeneity $\left(\mathrm{I}^{2}: 99 \%\right)$ and mean difference $(1.20 \mathrm{~mm}, \mathrm{P}<0.20)$.

\section{Financial support}

This research did not receive any specific grant from funding agencies in the public, commercial, or not-for-profit sectors.

\section{Conflict of interest}

The authors declare that there are no conflicts of interest.

\section{Acknowledgements}

None

\section{References}

Ainamo, J., \& Bay, I. (1975). Problems and proposals for recording gingivitis and plaque. International Dental Journal, 25, 229-235.

Ainamo, J., Barmes, D., Beagrie, G., Cutress, T., Martin, J., \& Sardo-Infirri, J. (1982). Development of the World Health Organization (WHO) community periodontal index of treatment needs (CPITN)? International Dental Journal, 32(3), 281-291.

Annibali, S., Bignozzi, I., Cristalli, M. P., Graziani, F., La Monaca, G., \& Polimeni, A. (2012). Peri-implant marginal bone level: A systematic review and meta-analysis of studies comparing platform switching versus conventionally restored implants. Journal of Clinical Periodontology, 39(11), 1097-1113. http://dx.doi.org/10.1111/j. 1600-051X.2012.01930.x.

Araújo, M. M., Martins, C. C., Costa, L. C., Cota, L. O., Faria, R. L., Cunha, F. A., et al. (2016). Association between depression and periodontitis: A systematic review and meta-analysis. Journal Clinical Periodontology, 43(3), 216-228. http://dx.doi.org/10. 1111/jcpe.12510.

Atieh, M. A., Ibrahim, H. M., \& Atieh, A. H. (2010). Platform switching for marginal bone 
preservation around dental implants: A systematic review and meta-analysis. Journal of Periodontology, 81(10), 1350-1366. http://dx.doi.org/10.1902/jop.2010.100232.

Bramanti, E., Bramanti, A., Matacena, G., Bramanti, P., Rizzi, A., \& Cicciù, M. (2015) Clinical evaluation of the oral health status in vascular-type dementia patients. A case-control study. Minerva Stomatologica, 64(4), 167-175.

Cestari, J. A., Fabri, G. M., Kalil, J., Nitrini, R., Jacob-Filho, W., Tesseroli de Siqueira, J. T., et al. (2016). Oral infections and cytokine levels in patients with Alzheimer's disease and mild cognitive impairment compared with controls. Journal of Alzheimer's Disease, 54(2), 845. http://dx.doi.org/10.3233/JAD-160212.

Chalmers, J. M., Carter, K. D., \& Spencer, A. J. (2003). Oral diseases and conditions in community-living older adults with and without dementia. Special Care in Dentistry, 23(1), 7-17.

Chen, X., Shuman, S. K., Hodges, J. S., Gatewood, L. C., \& Xu, J. (2010). Patterns of tooth loss in older adults with and without dementia: A retrospective study based on a minnesota cohort. Journal of the American Geriatrics Society, 58(12), 2300-2307. http://dx.doi.org/10.1111/j.1532-5415.2010.03192.x.

Chen, X., Clark, J. J., \& Naorungroj, S. (2013). Oral health in nursing home residents with different cognitive statuses. Gerodontology, 30(1), 49-60. http://dx.doi.org/10.1111/ j.1741-2358.2012.00644.x.

Chen, C. K., Wu, Y. T., \& Chang, Y. C. (2017). Association between chronic periodontitis and the risk of Alzheimer's disease: A retrospective, population-based, matched-cohort study. Alzheimer's Research \& Therapy, 9(1), http://dx.doi.org/10.1186/s13195017-0282-6 [56].

Chu, C. H., Ng, A., Chau, A. M., \& Lo, E. C. (2015). Oral health status of elderly Chinese with dementia in Hong Kong. Oral Health and Preventive Dentistry, 13(1), http://dx. doi.org/10.3290/j.ohpd.a32343 [51-57.

de Almeida, J. M., Matheus, H. R., Rodrigues Gusman, D. J., Faleiros, P. L., Januário de Araújo, N., \& Noronha Novaes, V. C. (2017). Effectiveness of mechanical debridement combined with adjunctive therapies for nonsurgical treatment of periimplantitis: A systematic review. Implant Dentistry, 26(1), 137-144. http://dx.doi.org/10.1097/ID. 0000000000000469.

De Souza Rolim, T., Fabri, G. M., Nitrini, R., Anghinah, R., Teixeira, M. J., de Siqueira, J. T., et al. (2014). Oral infections and orofacial pain in alzheimer's disease: A casecontrol study. Journal of Alzheimer's Disease, 38(4), 823-829. http://dx.doi.org/10. 3233/JAD-131283.

Dementia A public health priority 2012. World Health Organization \& Alzheimer's Disease International. Available at: http://www.who.int/mental health/ publications/dementia report 2012/en/. Accessed 21 November 2016

Dhingra, K., \& Vandana, K. L. (2011). Indices for measuring periodontitis: A literature review. International Dental Journal, 61(2), 76-84. http://dx.doi.org/10.1111/j.1875 595X.2011.00018.x.

Fereshtehnejad, S. M., Garcia-Ptacek, S., Religa, D., Holmer, J., Buhlin, K., Eriksdotter, M., et al. (2017). Dental care utilization in patients with different types of dementia A longitudinal nationwide study of 58,037 individuals. Alzheimer's \& Dementia, 1552-5260(17), 30233-30239. http://dx.doi.org/10.1016/j.jalz.2017.05.004.

Gil-Montoya, J. A., Sanchez-Lara, I., Carnero-Pardo, C., Fornieles, F., Montes, J., Vilchez, R., et al. (2015). Is periodontitis a risk factor for cognitive impairment and dementia? A case-control study. Journal of Periodontology, 86(2), 244-253. http://dx.doi.org/10. 1902/jop.2014.140340.

Gil-Montoya, J. A., Barrios, R., Santana, S., Sanchez-Lara, I., Pardo, C. C., Fornieles-Rubio, F., et al. (2017). Association between periodontitis and amyloid- $\beta$ peptide in elderly people with and without cognitive impairment. Journal of Periodontology, 1-10. http://dx.doi.org/10.1902/jop.2017.170071 Epub ahead of print.

Gil-Montoya, J. A., Sánchez-Lara, I., Carnero-Pardo, C., Fornieles-Rubio, F., Montes, J. Barrios, R., et al. (2017). Oral hygiene in the elderly with different degrees of cognitive impairment and dementia. Journal of the American Geriatrics Society, 65(3), 642-647. http://dx.doi.org/10.1111/jgs.14697.

Haffajee, A. D., \& Socransky, S. S. (1986). Attachment level changes in destructive periodontal diseases. Journal of Clinical Periodontology, 13(5), 461-475. http://dx.doi. org/10.1111/j.1600-051X.1986.tb01491.x.

Hatipoglu, M. G., Kabay, S. C., \& Güven, G. (2011). The clinical evaluation of the oral status in Alzheimer-type dementia patients. Gerodontology, 28(4), 302-306. http:// dx.doi.org/10.1111/j.1741-2358.2010.00401.x.

Higgins, J. P., \& Thompson, S. G. (2002). Quantifying heterogeneity in a meta-analysis. Statistics in Medicine, 21(11), 1539-1558.

Joss, A., Adler, R., \& Lang, N. P. (1994). Bleeding on probing. A parameter for monitoring periodontal conditions in clinical practice. Journal of Clinical Periodontology, tb00737. http://dx.doi.org/10.1111/j.1600-051X.1994.

Kamer, A. R., Craig, R. G., Pirraglia, E., Dasanayake, A. P., Norman, R. G., Boylan, R. J., et al. (2009). TNF-alpha and antibodies to periodontal bacteria discriminate between Alzheimer's disease patients and normal subjects. Journal of Neuroimmunology, 216(1-2), 92-97. http://dx.doi.org/10.1016/j.jneuroim.2009.08.013.

Kim, J. M., Stewart, R., Prince, M., Kim, S. W., Yang, S. J., Shin, I. S., et al. (2007). Dental health, nutritional status and recent-onset dementia in a Korean community population. International Journal of Geriatric Psychiatry, 22(9) [850-805.

Kurgan, S., \& Kantarci, A. (2017). Molecular basis for immunohistochemical and inflammatory changes during progression of gingivitis to periodontitis. Periodontology, 2000. http://dx.doi.org/10.1111/prd.12146.

Leask, A., Holmes, A., Black, C. M., \& Abraham, D. J. (2003). Connective tissue growth factor gene regulation. Requirements for its induction by transforming growth factorbeta 2 in fibroblasts. The Journal of Biological Chemistry, 278(15), 13008-13015. http://dx.doi.org/10.1074/jbc.M210366200.
Lee, Y. L., Hu, H. Y., Huang, L. Y., Chou, P., \& Chu, D. (2017). Periodontal disease associated with higher risk of dementia: Population-Based cohort study in Taiwan. Journal of the American Geriatrics Society, 65(9), 1975-1980. http://dx.doi.org/10. 1111 /jgs.14944.

Lee, Y. T., Lee, H. C., Hu, C. J., Huang, L. K., Chao, S. P., Lin, C. P., et al. (2017) Periodontitis as a modifiable risk factor for dementia: A nationwide population-Based cohort study. Journal of the American Geriatrics Society, 65(2), 301-305. http://dx.doi. org/10.1111/jgs.14449.

Li, W., Ma, D., Liu, M., Liu, H., Feng, S., Hao, Z., et al. (2008). Association between metabolic syndrome and risk of stroke: A meta-analysis of cohort studies. Cerebrovascular Diseases, 25(6), 539-547. http://dx.doi.org/10.1159/000131672.

Listgarten, M. A. (1980). Periodontal probing: What does it mean? Journal of Clinical Periodontology, 7(3), 165-176. http://dx.doi.org/10.1902/jop.1996.67.6.555.

Luo, J., Wu, B., Zhao, Q., Guo, Q., Meng, H., Yu, L., et al. (2015). Association between tooth loss and cognitive function among 3063 Chinese older adults: A communitybased study. Public Library of Science, 10(3), http://dx.doi.org/10.1371/journal.pone. 0120986 [e0120986.].

Martande, S. S., Pradeep, A. R., Singh, S. P., Kumari, M., Suke, D. K., Raju, A. P., et al. (2014). Periodontal health condition in patients with Alzheimer's disease. American Journal of Alzheimer's Disease and Other Dementias, 29(6), 498-502. http://dx.doi.org/ $10.1177 / 1533317514549650$.

Miller, S. A., \& Forrest, J. L. (2001). Enhancing your practice through evidence-based decision making: PICO, learning how to ask good questions. The Journal of Evidence Based Dental Practice, 1(2), 136-141.

Moher, D., Liberati, A., Tetzlaff, J., Altman, D. G., \& PRISMA, G. (2010). Preferred reporting items for systematic reviews and meta-analyses: The PRISMA statement. International Journal of Surgery, 8(5), 336-341. http://dx.doi.org/10.1016/j.ijsu. 2010.02.007.

Newbrun, E. (1996). Indices to measure gingival bleeding. Journal of Periodontology, 67(6), 555-561.

Noble, J. M., Manly, J. J., Schupf, N., Tang, M. X., \& Luchsinger, J. A. (2012). Type 2 diabetes and ethnic disparities in cognitive impairment. Ethnicity \& Disease, 22(1), $38-44$.

Noble, J. M., Scarmeas, N., Celenti, R. S., Elkind, M. S., Wright, C. B., Schupf, N., et al (2014). Serum IgG antibody levels to periodontal microbiota are associated with incident Alzheimer disease. Public Library of Science, 9(12), http://dx.doi.org/10. 1371/journal.pone.0114959 [e114959].

Noble, J., Burkett, S., Cheng, B., Chen, Y., Shariff, J., Celenti, R., et al. (2017). Crosssectional associations between clinical and serological evidence of periodontal disease and cognitive impairment in a multi-ethnic elderly population. Neurology, 88(Suppl. (1)), 16.

Okamoto, N., Morikawa, M., Okamoto, K., Habu, N., Iwamoto, J., Tomioka, K., et al. (2010). Relationship of tooth loss to mild memory impairment and cognitive impairment: Findings from the Fujiwara-kyo study. Behavioral and Brain Functions, 6, 77. http://dx.doi.org/10.1186/1744-9081-6-77.

Pazos, P., Leira, Y., Domínguez, C., Pías-Peleteiro, J. M., Blanco, J., \& Aldrey, J. M. (2016). Association between periodontal disease and dementia: A literature review. Neurologia, S0213-S4853(16), http://dx.doi.org/10.1016/j.nrl.2016.07.013 30178-30175.

Rai, B., Kaur, J., \& Anand, S. C. (2012). Possible relationship between periodontitis and dementia in a North Indian old age population: A pilot study. Gerodontology, 29(2), e200-e205. http://dx.doi.org/10.1111/j.1741-2358.2010.00441.x.

Schroeder, H. E., Munzel-Pedrazzoli, S., \& Page, R. (1973). Correlated morphometric and biochemical analysis of gingival tissue in early chronic gingivitis in man. Archives of Oral Biology, 18, 899-923.

Singh, K. (2016). Correlation of mild cognitive impairment, periodontitis and stroke in elderly Indian population. Alzheimers \& Dementia, 12(Suppl. (7)) P902.

Sochocka, M., Sobczyński, M., Sender-Janeczek, A., Zwolińska, K., Błachowicz, O. Tomczyk, T., et al. (2017a). Association between periodontal health status and cognitive abilities. The role of cytokine profile and systemic inflammation. Current Alzheimer Research. http://dx.doi.org/10.2174/1567205014666170316163340 Epub ahead of print.

Sochocka, M., Sobczyński, M., Sender-Janeczek, A., Zwolińska, K., Błachowicz, O., Tomczyk, T., et al. (2017b). Cognitive impairment, periodontal disease and systemic inflammation-is there a link? Neurodegenerative Disease Management, 17(Suppl. 1), 1065.

Sparks Stein, P., Steffen, M. J., Smith, C., Jicha, G., Ebersole, J. L., Abner, E., et al. (2012). Serum antibodies to periodontal pathogens are a risk factor for Alzheimer's disease Alzheimer's \& Dementia, 8(3), 196-203. http://dx.doi.org/10.1016/j.jalz.2011.04. 006.

Stewart, R., Stenman, U., Hakeberg, M., Hägglin, C., Gustafson, D., \& Skoog, I. (2015). Associations between oral health and risk of dementia in a 37-year follow-up study: The prospective population study of women in Gothenburg. Journal of the American Geriatrics Society, 63(1), 100-105. http://dx.doi.org/10.1111/jgs.13194.

Syrjälä, A. M., Ylöstalo, P., Ruoppi, P., Komulainen, K., Hartikainen, S., Sulkava, R., et al. (2012). Dementia and oral health among subjects aged 75 years or older. Gerodontology, 29(1), 36-42. http://dx.doi.org/10.1111/j.1741-2358.2010.00396.x.

Takeuchi, K., Ohara, T., Furuta, M., Takeshita, T., Shibata, Y., Hata, J., et al. (2017). Tooth loss and risk of dementia in the community: The hisayama study. Journal of the American Geriatrics Society, 65(5), http://dx.doi.org/10.1111/jgs.14791 [e95-e100].

Welch, V., Petticrew, M., Tugwell, P., Moher, D., O'Neill, J., Waters, E., et al. (2012). PRISMA-Equity Bellagio group. PRISMA-Equity 2012 extension: Reporting guidelines 
for systematic reviews with a focus on health equity. PLoS Medicine, 9(10), e1001333. http://dx.doi.org/10.1371/journal.pmed.1001333.

Wells G., Shea B., O'Connell D., Peterson J., Welch V., Losos M., Tugwell P. (2011) The Newcastle-Ottawa Scale (NOS) for assessing the quality of nonrandomised studies in metaanalyses. URL http://www.ohri.ca/programs/clinical_epidemiology/oxford.asp [Accessed on 20 November 2016].

World Alzheimer Report 2009. Alzheimer's Disease International. Available at: https:// www.alz.co.uk/research/files/WorldAlzheimerReport.pdf. Accessed 22 November, 2016.

World Health Organization 2005. Periodontal country profiles. Available at: http://www. who.int/oral_health/databases/niigata/en/.

Wu, B., Fillenbaum, G. G., Plassman, B. L., \& Guo, L. (2016). Association between oral health and cognitive status: A systematic review. Journal of the American Geriatrics Society, 64(4), 739-751. http://dx.doi.org/10.1111/jgs.14036.
You, Y. S., Qu, N. B., \& Yu, X. N. (2016). Alcohol consumption and dry eye syndrome: A meta-analysis. International Journal of Ophthalmology, 9(10), 1487-1492.

Zenthöfer, A., Baumgart, D., Cabrera, T., Rammelsberg, P., Schröder, J., Corcodel, N., et al. (2017). Poor dental hygiene and periodontal health in nursing home resident with dementia: An observational study. Odontology, 105(2), 203-213. http://dx.doi. org /10.1007/s10266-016-0246-5.

Zenthöfer, A., Cabrera, T., Rammelsberg, P., \& Hassel, A. J. (2016). Improving oral health of institutionalized older people with diagnosed dementia. Aging and Mental Health, 20(3), 303-308. http://dx.doi.org/10.1080/13607863.2015.1008986.

Zenthöfer, A., Meyer-Kühling, I., Hufeland, A. L., Schröder, J., Cabrera, T., Baumgart, D., et al. (2016). Carers' education improves oral health of older people suffering from dementia - results of an intervention study. Clinical Interventions in Aging, 11, 1755-1762. 